\title{
Identification of excreted iron superoxide dismutase for the diagnosis of Phtytomonas
}

\author{
Clotilde Marín, Isabel Rodríguez-González, Manuel Sánchez-Moreno/ ${ }^{+}$
}

\author{
Instituto de Biotecnología, Departamento de Parasitología, Facultad de Ciencias, Universidad de Granada, \\ C/ Severo Ochoa s/n, 18071 Granada, España
}

\begin{abstract}
An excreted iron superoxide dismutase (FeSODe) of pI 3.6 with a molecular weight of 28-30 $\mathrm{kDa}$ was detected in the in vitro culture of Phytomonas isolated from Euphorbia characias (SODeCHA) and from Lycopersicon esculentum (SODeTOM), in Grace's medium without serum. These FeSODe excreted into the medium had immunogenic capacity: the positivity of the anti-SODeCHA serum persisted to a dilution of 1/30,000, and for the anti-SODeTOM to 1/ 10,000 by Western blot. In addition, cross reaction was detected between the anti-SODe serum of Phytomonas isolated from E. characias against SODeTOM, and the anti-SODe serum from L. esculentum with SODeCHA. This characteristic offers the possibility of its use to diagnose plant trypanosomatids. The validation of the test was confirmed by experimental inoculation of tomato fruits with Phytomonas isolated from $\mathrm{L}$. esculentum. At 7, 10, 15, and 21 days post infection, it was possible to detect the presence of the parasites with the anti-SODe serum of Phytomonas isolated from $\mathrm{L}$. esculentum at a dilution of 1/250. These serological results were confirmed by visualization of the parasites by optical microscopy. The data of this study confirm that the SOD is sufficient to identify a trypanosomatid isolated from plants as belonging to the genus Phytomonas.
\end{abstract}

Key words: superoxide dismutase - immunogenicity - molecular tool - Phytomonas spp.

Trypanosomatids belonging to the genus Phytomonas are fairly common in the latex, phloem, fruit sap, seed albumen, and even in the nectar of many plant families. Also, their world distribution is widespread. They are etiological agents in devastating crop epiphytotics (coconut, oil palm, coffee, and manioc) but they also parasitize many plants without apparent pathogenicity (Dollet 1984).

Probably more than one genus of Trypanosomatidae is represented among plant parasites. Therefore, both the study of the circulation of parasites as well as the demonstration of infection in hosts require a precise identification of trypanosomatids. The distinctions between them is difficult on a morphological basis. Attempts to overcome this difficulty prompted the testing of various serological, biochemical, and molecular methods, but none to date have been very successful for genus distinction (Camargo 1999). In recent years, new polymerase chain reaction method for the amplification of the SL sequence have been successfully developed, and more recently new molecular markers have become available to help in the identification (Serrano et al. 1999, Dollet et al. 2001, Marín et al. 2004a).

Superoxide dismutases (SODs, EC 1.15.1.1) are metalloproteins that occur ubiquitously in nature and efficiently dismutase the superoxide anion into oxygen and hydrogen peroxide. SODs are assigned to different families,

Finacial support: ATP 2002/03: Circulation of Trypanosomatidae Project (CIRAD, France), grant BIO-2000-1429 (University of Granada, Spain)

Corresponding author: msanchem@ugr.es

Received 16 March 2006

Accepted 21 June 2006 based on their metal cofactors - iron (FeSOD), manganese $(\mathrm{MnSOD})$ and copper-zinc $(\mathrm{Cu} / \mathrm{ZnSOD})$ - which also differ in their location: cytosol, cell organelles, and even cell excretions. Thus, the $\mathrm{Cu} / \mathrm{ZnSODs}$ appear primarily in the cytosol of eukaryotic cells; the MnSODS in the mitochondrial matrix; and FeSODs in the cytosol of plant cells as well as in some free-living protozoa and protozoan parasites (Fridovich 1995).

SOD activity has been detected in the main species belonging to the family Trypanosomatidae, in Trypanosoma cruzi (Ismail et al. 1997), in T. brucei brucei (Kabiri $\&$ Steverding 2001), in several species of the genus Leishmania (Ismail et al. 1994), in some lower trypanosomatids, and in plant trypanosomes (Quesada et al. 2001, Marín et al. 2004a,b). SODs from protozoan parasites are considered virulence factors that protect the parasites from the attack of the host cells by the action both of oxidant and anti-inflammatory agents (Paramchuck et al. 1997), and can even confer immunological capacities (Pérez-Fuentes et al. 2003). In general, all the parasitic protozoa studied to date have only FeSOD (Marín et al. 2004a,b).

Extracellular SODs (EcCu/ZnSODs) have been evidenced in invertebrates, in several species of nematodes, such as, Brugia pahangi (Tang et al. 1994), Onchocerca volvulus (James et al. 1994), and Caenorhabditis elegans (Fujii et al. 1998) and the crustacean Callinectes sapidus (Brouwer et al. 2003). Recently, Villagrán et al. (2005) have shown the presence of a iron SODe in T. cruzi. Also, in mammals, several researchers have studied extracellular SOD (EC-SOD), which is the major extracellular antioxidant enzyme and plays a critical role in the pathogenesis of a variety of pulmonary, neurological, and cardiovascular diseases (Suliman et al. 2001, Fukai et al. 2002).

In a previous paper, we reported in a digitonin-titration experiment that $20 \%$ of the SOD activity of Phy- 
tomonas sp. was not solubilized even at high digitonin concentrations, indicating that part of the SOD could be associated with membranes or even excreted by the parasite (Marín et al. 2004b). Therefore, in the present study, we show the existence of a SOD excreted into the supernatant from the culture of Phytomonas isolated from $\mathrm{Eu}$ phorbia characias, and Phytomonas isolated from $L y$ copersicon esculentum, cultured in Grace's medium without serum. Also, we have studied the usefulness of excreted SOD in the identification and diagnosis of Phytomonas infections using Western blot in the identification

\section{MATERIALS AND METHODS}

Parasites and SOD excreted fraction extraction Phytomonas isolated from latex vessels of $E$. characias (Dollet 1984) and from L. esculentum (tomato) in Spain (Sánchez-Moreno et al. 1995), were grown in axenic Grace's medium (Sigma) supplemented with $10 \%$ heat inactivated foetal bovine serum at $28^{\circ} \mathrm{C}$. Cells at the exponential growth phase were collected by centrifugation $(1500 \times \mathrm{g}$ for $5 \mathrm{~min}$ at room temperature). The pellet of cells was washed twice in Grace's medium without serum, and cells were counted, distributed into aliquots of $5 \times 10^{9}$ parasites $/ \mathrm{ml}$ in Grace's medium without serum, and grown for $24 \mathrm{~h}$. The supernatant was collected by centrifugation at $1500 \times \mathrm{g}$ for $10 \mathrm{~min}$, passed through a $0.45 \mu \mathrm{m}$ pore size filter, and then precipitated with ammonium sulphate at $35-85 \%$. This was centrifuged $\left(9000 \times \mathrm{g}\right.$ for $20 \mathrm{~min}$ at $\left.4{ }^{\circ} \mathrm{C}\right)$, redissolved in $2.5 \mathrm{ml}$ of $50 \mathrm{mM}$ potassium phosphate buffer ( $\mathrm{pH} 7.8$ ), and afterwards desalted by buffer exchange in a Sephadex G-25 column (Pharmacia, PD 10), previously balanced with the potassium buffer, bringing it to a final volume of $3.5-\mathrm{ml}$. Thus, we obtained the SODe fraction from Phytomonas isolated from E. characias (SODeCHA) and SODe from Phytomonas isolated from L. esculentum (SODeTOM). These fractions were finally concentrated by ultrafiltration in Centriprep-10 tubes (Amicon) at $3000 \times \mathrm{g}$ to a final concentration of $2 \mathrm{mg} / \mathrm{ml}$. The protein content was determined in all fractions using the Bio-Rad test, based on the Bradford method (Bradford 1976), with BSA as a standard. For the extraction and purification of the P85 fraction, we followed the protocol described by Marín et al. (2004b).

Determination of molecular weights and isoelectric point of SODe - Apparent molecular weights of the semipurified enzymes (SODeCHA and SODeTOM) were determined by the separation of $7 \mu \mathrm{g} /$ lane on $20 \%$ homogeneous Native-PAGE gel in the Phast System (Amersham Pharmacia Biotech, Uppsala, Sweden). The isoelectric points were determined in the Phast System $(7 \mu \mathrm{g} / \mathrm{lane})$ in polyacrylamide Phast gel pI 3-9 as described Bécuwe et al. (1996). Proteins markers for molecular weights and pI were provided by Pharmacia (Uppsala, Sweden). The SOD activity on the gels was visualized by staining, following Beyer and Fridovich (1976), and for protein markers the lanes were stained with silver nitrate and coomassie blue, as described by Phast System handbook.

Polyclonal serum - To obtain the specific antibodies against the fractions SODeCHA and SODeTOM, we im- munized two female 4-week-old Balb-C mice (concentration of proteins of $2 \mathrm{mg} / \mathrm{ml}$ ). These fractions were separated by electrophoresis of IEF 3-9 in polyacrylamide gels as described elsewhere (Marín et al. 2004a). In this way, we obtained the anti-SODeCHA and anti-SODeTOM sera. In addition, the serum from the mouse that had not been immunised with the antigen fraction (control serum) was collected.

Experimental infection of tomatoes - Isolated tomato were infected by needle inoculation in two zones, with $5 \times$ $10^{5}$ culture forms of Phytomonas isolated from L. esculentum in $50 \mu \mathrm{l}$. As controls, three tomatoes were inoculated with the same volume of axenic Grace's medium supplemented with $10 \%$ heat-inactivated foetal bovine serum. At 7, 10, 15, and 21 days post-inoculation, three tomatoes, controls and infected, were processed to obtain the extracts for later studies. After the inoculation zones were collected, smears fixed with methanol and stained by Giemsa were used for light microscopy. Afterwards, the material was homogenized and centrifuged at $1500 \times \mathrm{g}$ for $10 \mathrm{~min}$. The supernatant was filtered, precipitated with ammonium sulphate, passed through a Sephadex G-25 column, and concentrated as described above for obtaining the SODe fraction. Also, the zones opposite the inoculation from infected tomatoes were processed the same way.

Western-blot analysis - For the polyclonal serum titration, we used the SODe fraction (SODeCHA and SODeTOM) as the antigen fraction. These were run on IEF 3-9 gels (protein concentration of proteins of $2 \mathrm{mg}$ / $\mathrm{ml}$ ), and afterwards transferred to nitrocellulose, for 30 min, as prescribed in the Phast-System manual. The membrane was blocked for $2 \mathrm{~h}$ at room temperature using $0.4 \%$ gelatine and $0.2 \%$ Tween 20 in PBS, followed by three washes in $0.1 \%$ Tween 20 in PBS (PBS-T). Next, the membrane was incubated for $2 \mathrm{~h}$ at room temperature, either with negative serum at a 1/100 dilution or with antiSODeCHA or anti-SODeTOM sera at dilutions of: 1/100, $1 / 500,1 / 1000,1 / 5000,1 / 10,000,1 / 20,000$, and $1 / 30,000$. After being washed as above, the membrane was further incubated for $2 \mathrm{~h}$ at room temperature with the second antibody, anti-mouse $\mathrm{IgG}(\mathrm{Fc}$ specific) peroxidase conjugate (Sigma ${ }^{\circledR}$ ) (dilution 1/1000). After washing, the substrate diaminobenzidine was added and the reaction stopped with several washes in distilled water (Marín et al. 2004a). For the cross reaction analyses, we followed the same protocol described above but with a dilution of the anti-SODeCHA and anti-SODeTOM sera of 1/5000.

Western-blot was also used to detect the SODeTOM in experimentally inoculated tomato fruits, as described above. For this, the extracts from the tomato of 7, 10, 15, and 21 days were used as the antigen fraction, as were the extracts of control tomatoes. The dilution of the antiSODeTOM serum of $1 / 250$ was used.

\section{RESULTS}

Isolation of an excreted FeSOD - When promastigote forms of Phytomonas isolated from E. characias and $L$. esculentum $\left(5 \times 10^{9}\right.$ parasites $)$ were cultured for $24 \mathrm{~h}$ in Grace's medium without serum and the cell-free superna- 
tant was collected, concentrated, separated by isoelectric focusing (pI 3-9) and 20\% homogeneous native-page and followed by SOD activity staining. We detected a single SOD band (Fig. 1A, lanes 2 and 4; Fig.1B, lanes 5 and 6), which we called SODeCHA and SODeTOM, respectively.

The isoelectric point of these bands of excreted SOD (SODeCHA and SODeTOM) was consistent with the isoelectric point (pI 3.6) of the SODII band detected in the partially purified fraction of Phytomonas promastigotes cultured under normal conditions (P85) (Fig. 1, lanes, 1 and 3).

The two isoenzymes excreted had very similar molecular weights, of approximately $28-30 \mathrm{kDa}$ (Fig. 1). In both cases, they were demonstrated to be FeSOD (data not shown).

Marker enzymes (pyruvate kinase and hexokinase) have confirmed that there was no lysis of the parasite during this culture period and that the presence of the SOD was due only to excretion by the parasite (data not shown).

Immunogenicity of the SODe - Polyclonal antibodies against the enzymes SODeCHA and SODeTOM were obtained from immunized Balb-C mice, and, by Western blot,

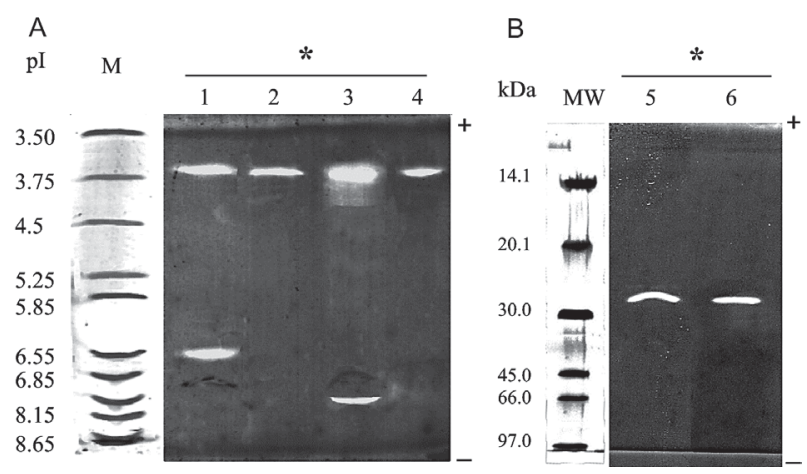

Fig. 1: determination of isoelectric point by isoelectrofocus pI 3-9 and molecular weight in $20 \%$ homogeneous native-PAGE electrophoresis. A: isoelectrofocus pI 3-9; B: $20 \%$ homogeneous nativePAGE electrophoresis. Lanes - 1, 2, and 5 of Phytomonas isolates from Euphorbia characias. 1: fraction P85; 2 and 5: fraction excreted; 3, 4, and 6 of Phytomonas isolated from Lycopersicon esculentum. 3: fraction P85; 4 and 6: fraction excreted; *: activity revealed according to the technique of Beyer and Fridovic (1987); M: marker proteins of $\mathrm{pI}$, stained with Coomassie Blue; MW: molecular-weight-marker protein, stained by silver nitrate. we demonstrated their immunogenic capacity (Fig. 2). With the control serum (Fig. 2, lines 1 and 9), the reaction was negative at a dilution of $1 / 100$, regardless of the antigen fraction. Meanwhile, in the case of the anti-SODe serum (Fig. 2; lanes 2-8 and lanes 10-16, respectively), the reactions proved positive up to a dilution of 1/30,000 for SODeCHA and 1/10,000 for SODe-TOM.

We determined whether there was any cross reaction between the polyclonal sera antiSODeCHA and SODeTOM, by Western blot (Fig. 3). There was cross reactivity between the sera to a dilution of $1 / 5000$.

Detection of SODeTOM in infected tomatoes - At 7 , 10,15 , and 21 days after infection, the inoculation zones and opposite ones were processed as described to be analyzed by Western blot. The tomato extracts were used as the antigen fraction against the anti-SODeTOM serum at the dilution of $1 / 250$, and, the presence of SODeTOM was detected (Fig. 4). In addition, not only did the inoculation zone prove positive, but on day 21 post-infection, we demonstrated the presence of these parasites in zones opposite the inoculation in the infected tomatoes (Fig. 4, line 5). Thus, we showed that there is dissemination of the parasites in the tomato.

These results were confirmed by direct examination of the parasite by light microscopy (Fig. 5). The flagellates

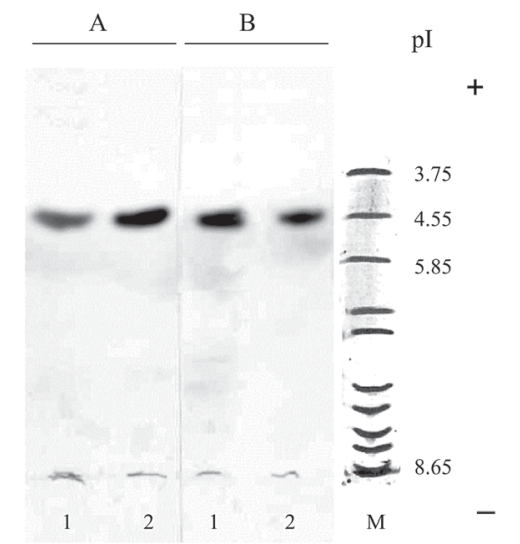

Fig. 3: cross reaction of polyclonal antiSODe sera against SODe by Western blot at dilution 1/5000. A: antiserum SODeTOM; B: antiserum SODeCHA; 1 SODeCHA and 2 SODeTOM; marker proteins of pI, stained with Coomassie Blue.

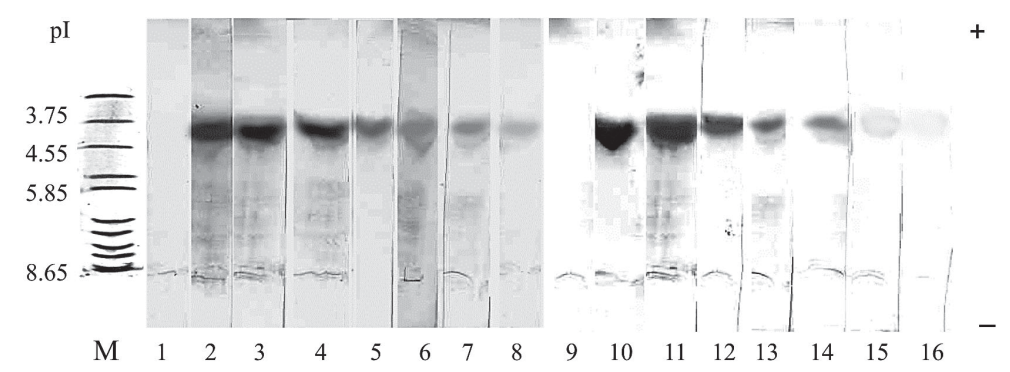

Fig. 2: titration of the polyclonal serum anti-SODeCHA and anti-SODeTOM by Western blot. Lanes - 1 and 9: control serum, dilution 1/100; 2-8: serum anti-SODeCHA; 10-16: serum anti-SODeTOM. Dilutions: 2 and 10, 1/100; 3 and 11, 1/500; 4 and 12, 1/1000; 5 and 13, 1/5000; 6 and 14, 1/10,000; 7 and 15, 1/20,000; and 8 and 16, 1/30,000; marker proteins of pI, stained with Coomassie Blue. 


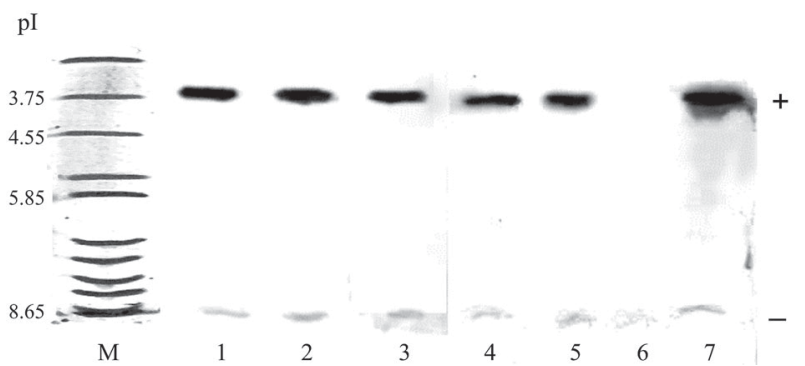

Fig. 4: Western blot of the polyclonal sera anti-SODeTOM at a dilution of $1 / 250$, against the infected tomato extract. Lanes - 1 : day $7 ; 2$ : day $10 ; 3$ : day $15 ; 4$ : day $21 ; 5$ : zone opposite the inoculation day 21; 6: tomato extract control; and 7: SODeTOM; marker proteins of $\mathrm{pI}$, stained with Coomassie Blue. detected presented the typical promastigote form, with a twisted body and in most cases with a large flagellum, although the smallest promastigote forms without flagella were also visible.

\section{DISCUSSION}

In previous studies, we have purified and characterized two isoenzymes (SODI and SODII) from Phytomonas isolated from E. characias (Marín et al. 2004a,b), confirming that one of these (SODII), with a pI 3.6, presented immunogenic properties.

Currently, the direct way to detect the presence of flagellates belonging to the genus Phytomonas in a plant is by observation with the light microscope. This makes the identification and the diagnosis difficult and at times
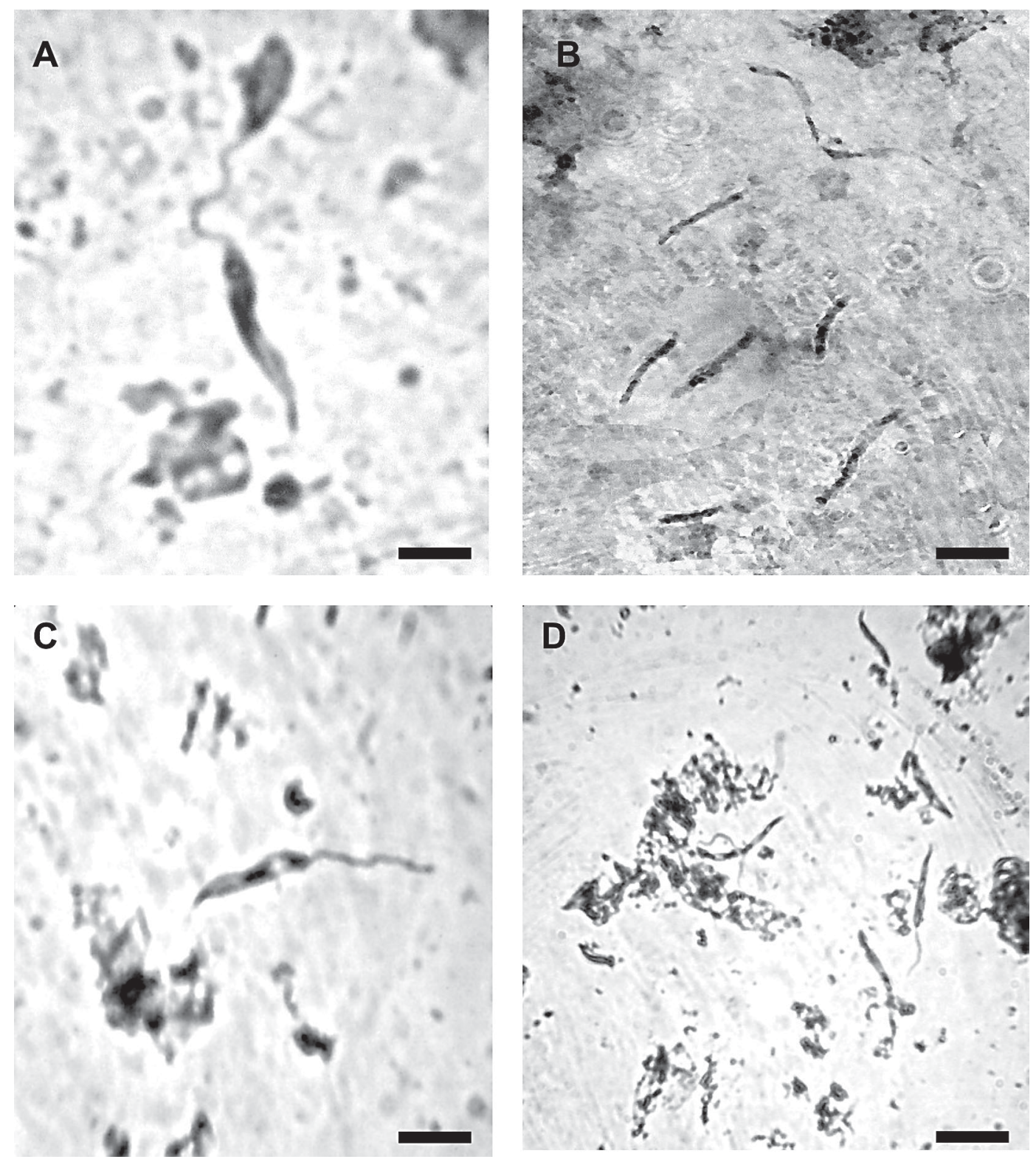

Fig. 5: detection of Phytomonas isolated from Lycopersicon esculentum in tomato by light microscopy. A: day 7; B: day 10; C: day 15; and D: day 21 . Bar $=1 \mu \mathrm{m}$. 
even impossible, because promastigote stages of Leptomonas, Herpetomonas, Crithidia, and Blastocrithidia also occur in Phytomonas vectors (Wallace et al. 1992, Camargo \& Wallace 1994, Podlipaev 2000), and have sometimes been detected in plant tissues. Thus, it becomes necessary to seek new methods to detect the parasite. Diverse authors have suggested that Western blot can be used as a complementary and alternative method for conventional serology tests (Ávila et al. 1993), and therefore may be useful in detecting and identifying parasitic protozoa using antigens from epimastigotes forms and SODe (Vissoci et al. 1998, Villagrán et al. 2005). Also, we have demonstrated that the SODII for its immunogenic characteristics is a excellent molecular marker of the genus Phytomonas, which could be applied to the different immunological techniques.

To our knowledge, this is the first evidence that members belonging to the genus Phytomonas excrete a FeSOD into the culture medium. These data confirm the findings of our earlier study (Marín et al. 2004a), showing that SODII would be formed in the cytosol and afterwards transported to the glycosomes, as occurs with other glycosomal enzymes (Parsons et al. 2001), and to the membranes, and part of this enzyme could even be secreted to the exterior by the parasite, as a defence mechanism of the parasite against toxic radicals generated by the host (Kabiri \& Steverding 2001).

One of the aim in the present work was to study the immunogenic properties of this FeSODe, for which we obtained polyclonal antibodies against SODeCHA and SODeTOM from immunized Balb-C mice. Western blot showed a positive reaction up to a dilution of $1 / 30,000$. This result indicates that, FeSODe is a highly immunogenic protein, opening the possibility of its use for the diagnosis of parasitism by species belonging to the genus Phytomonas.

To confirm the usefulness of this excreted protein (SODe) as a marker in Western-blot diagnosis, we performed an inoculation experiment on tomato fruits. The presence of Phytomonas was detected at 7, 10, 15, and 21 days after infection in the inoculation zones with the antiSODeTOM serum at the dilution of $1 / 250$, and also on day 21 in the zones opposite the inoculation. Hence, SODe is a specific and sensitive molecular tool and, used with Western blot, can be useful to the diagnosis of Phytomonas spp. The flagellates detected in the laboratory-infected tomatoes by light microscopy presented the typical promastigote form, although the smallest promastigote forms without flagella were also visible, these being very similar to others reported elsewhere (Jankevicius et al. 1989).

The data of this study together with those gathered recently in our laboratory (Marín et al. 2004a,b) confirm that the SOD (SODII or SODe) is sufficient to identify a trypanosomatid isolated from plants as belonging to the genus Phytomonas and to distinguish between a true Phytomonas and other trypanosomatids that can provoke transient infections in plants. Also, a certain amount of SOD is secreted by the parasites, which is highly immunogenic and by Western blot assay capable of detecting the presence of Phytomonas isolates from L. esculentum in crude preparations from tomato saps. Thus, we were able to detect this parasitism without the need for isolation, culture, or DNA extraction of flagellates. Of course, the validity of the SODe by Phytomonas spp. as a diagnostic tool needs to be continuously evaluated as new isolates and molecular data became available

\section{REFERENCES}

Ávila HA, Pereira JB, Thiermann O, Paiva E, Degrave W, Morel, C M, Simpson, L 1993. Detection of Trypanosoma cruzi in blood specimens of chronic chagasic patients by polymerase chain reaction amplification of kinetoplast minicircle DNA: comparison with serology and xenodiagnosis. J Clin Microbiol 21: 2421-2426

Bécuwe P, Gratepanche S, Fourmaux MN, Van Beeumen J, Samyn B, Mercereau-Puijalon O, Touzel JP, Slomianny C, Camus D, Dive D 1996. Characterization of iron-dependent endogenous superoxide dismutase of Plasmodium falciparum. Mol Biochem Parasitol 76: 125-134

Beyer WF, Fridovich I 1987. Assaying for superoxide dismutase activity: some large consequences of minor changes in conditions. Anal Biochem 161: 559-566.

Bradford MM 1976. A refined and sensitive method for the cuantification of microgram quantities of protein-dye binding. Anal Biochem 72: 248.

Brouwer M, Hoexum Brouwer T, Grater W, Brown-Peterson N 2003. Replacement of a cytosolic copper/zinc superoxide dismutase by a novel cytosolic manganese superoxide dismutase in crustaceans that use copper (haemocyanin) for oxygen transport. Biochem J 374: 219-228.

Camargo EP 1999. Phytomonas and other trypanosomatid parasites of plants and fruit. Adv Parasitol 42: 29-112.

Camargo EP, Wallace G 1994. Vectors of plant parasites of the genus Phytomonas (Protozoa, Zoomastigophore, Kinetoplastida). In KF Harris, Advances in Disease Vector Research, vol. 10, Academic Press, New York, p. 333-359.

Dollet M, Sturm NR, Campbell DA 2001. The spliced leader RNA gene array in phloem-restricted plant trypanosomatids (Phytomonas) partitions into two major groupings: epidemiological implications. Parasitology 122: 289-297.

Dollet M 1984. Plant trypanosomes case study. In DL Hawksworth, Identification and Characterization of Pest Organism, CAB Interantional, Oxford, p. 415-426.

Fridovich I 1995. Superoxide radical and superoxide dismutases. Annu Rev Biochem 64: 97-112.

Fujii M, Ishii N, Joguchi A, Yasuda K, Ayusawa D 1998. A novel superoxide dismutase gene encoding membranebound and extracellular isoforms by alternative splicing in Caenorhabditis elegans. DNA Res 5: 25-30.

Fukai T, Folz RJ, Landmesser U, Harrison DG 2002. Extracellular superoxide dismutase and cardiovascular disease. Cardiovasc Res 55: 239-249.

Ismail SO, Paramchuk W, Yasir A, Skeiky YA, Reed SG, Bathia A, Gedamu L 1997. Molecular cloning and characterization of two iron superoxide dismutase cDNAs from Trypanosoma cruzi. Mol Biochem Parasitol 86: 187-197.

Ismail SO, Skeiky YA, Bhatia A, Omara-Opyene LA, Gedamu L 1994. Molecular cloning, characterization, and expresión in E. coli of iron superoxide dismutase cDNA from Leish- 
mania donovani chagasi. Infect Immun 62: 657-664.

James ER, McLean DC, Perler F 1994. Molecular cloning of an Onchocerca volvulus extracellular $\mathrm{Cu}-\mathrm{Zn}$ superoxide dismutase. Infect Immun 62: 713-716.

Jankevicius JV, Jankevicius SI, Campaner M, Conchon I, Maeda LA, Teixeira MMG, Freymuller E, Camargo E 1989. Life cycle and culturing of Phytomonas serpens (Gibbs), a trypanosomatid parasite of tomatoes. J Protozool 36 : 265-271.

Kabiri M, Steverding D 2001. Identification of a developmentally regulated iron superoxide dismutase of Trypanosome brucei. Biochem J 360: 173-177.

Marín C, Hitos AB, Rodríguez-González I, Dollet M, SánchezMoreno M 2004a. Phytomonas Iron Superoxide dismutase: A possible molecular marker. FEMS Microbiol Letters 234: 69-74.

Marín C, Rodríguez-González I, Hitos AB, Rosales MJ, Dollet M, Sánchez-Moreno M 2004b. Purification and characterization of two iron superoxide dismutases of Phytomonas sp. isolated from Euphorbia characias (plant trypanosomatids). Parasitology 129: 79-86.

Paramchuck WJ, Ismail SO, Bhatia A, Gedamu L 1997. Cloning, characterization and overexpression of two iron superoxide dismutase cDNAs from Leishmania chagasi: role in pathogenesis. Mol. Biochem Parasitol 90: 203-221.

Parsons M, Furuya T, Pal S, Kessler P 2001. Biogenesis and function of peroxisomes and glycosomes. Mol Biochem Parasitol 115: 19-28.

Pérez-Fuentes R, Guégan JF, Barnabé C, López-Colombo A, Salgado-Rosas H, Torres-Rasgado E, Briones B, RomeroDiaz M, Ramos-Jiménez J, Sánchez-Guillén MC 2003. Severtity of chronic Chagas disease is associated with citokine/antioxidant imbalance in chronically infected individuals. Int J Parasitol 33: 293-299.

Podlipaev S 2000. Insect trypanosomatids: the need to know more. Mem Inst Oswaldo Cruz 95: 517-522.
Quesada JM, Entrala E, Fernandez-Ramos C, Marin C, SanchezMoreno M. 2001. Phytomonas spp: superoxide dismutase in plant trypanosomes. Mol Biochem Parasitol 115: 123127.

Sánchez-Moreno M, Fernández-Becerra C, Mascaró C, Rosales MJ, Dollet M, Osuna A 1995. Isolation, in vitro culture, ultrastructure study and characterization by lectin-agglutination tests of Phytomonas isolated from tomatoes (Lycopersicon esculentum) and cherimoyas (Annona cherimolia) in southeastern Spain. Parasitol Res 81: 575581.

Serrano MG, Numes LR, Campaner M, Buck GA, Camargo EP, Teixeira MMG 1999. Trypanosomatidae: Phytomonas detection in plants phytophagous insects by PCR amplification of a genus-specific sequence of the Splice Leader gene. Exp Parasitol 91: 268-279.

Suliman HB, Ryan LK, Bioshop L, Folz RJ 2001. Prevention of influenza-induced lung injury in mice overexpressing extracellular superoxide dismutase. Am J Physiol Lung Cell Mol Physiol 280: 169-178.

Tang L, Ou X, Henkle-Duhrsen K, Selkirk ME 1994. Extracellular and cytoplasmic $\mathrm{CuZn}$ superoxide dismutases from Brugia lymphatic filarial nematode parasites, Infect Immun 62: 961-967.

Villagrán ME, Marín C, Rodríguez-González I, De Diego JA, Sánchez- Moreno M 2005. An iron superoxide dismutase (Fesode) excreted by Trypanosoma cruzi useful in the diagnosis of Chagas disease: seroprevalence of this infection in rural zones of the State of Queretaro (Mexico). Am J Trop Med Hyg 73: 510-516.

Vissoci Reiche, EM, Cavazzana M, Okamura H, Tagata EL, Jankevicius SI, Jankevicius JV 1998. Evaluation of the Western blot in the confirmatory serologic diagnosis of Chagas disease. Am J Trop Med Hyg 59: 750-756.

Wallace FG, Roitman I, Camargo EP 1992. Trypanosomatids of plants. In JP Kreir, LR Baker (eds), Parasitic Protozoa, Academic Press, New York, p. 55-85. 